\title{
Gestão do conhecimento: constituição do campo, temas e perspectivas de pesquisas
}

\author{
Knowledge management: the field's constitution, \\ themes, and research perspectives
}

Hilka Pelizza Vier MACHADO' (D) 0000-0002-2554-0025

Maria Ligia Ganacim Granado Rodrigues ELIAS'1 (D) 0000-0003-3645-9131

\begin{abstract}
Resumo
Esta pesquisa visa identificar e analisar temas de pesquisas em Gestão do Conhecimento, a fim de compreender como tem se constituído esse campo científico e quais as perspectivas de pesquisas. Para o desenvolvimento deste trabalho, realizou-se uma revisão sistemática da literatura a partir de estudos bibliométricos em Gestão do Conhecimento publicados no período de 2008 a 2018. Os dados foram agrupados em oito temas: natureza do conhecimento, dimensões sociais, dimensões cognitivas e tecnológicas do conhecimento, espectro do conhecimento, contextos, áreas de interfaces e estudos voltados ao desenvolvimento do campo cientííco. O estudo oferece um panorama temático do campo da Gestão do Conhecimento, apontando para a necessidade de que sejam abordados novos temas, atentos aos desafios comunicacionais contemporâneos. Como contribuições práticas, os resultados podem auxiliar pesquisadores, profissionais e estudantes que pretendam se inserir no campo. Os resultados fornecem também pistas para organizadores de conferências, ao apontar temas para discussões, e para editores científicos no sentido de orientar futuras chamadas de artigos.
\end{abstract}

Palavras-chave: Bibliométrico. Gestão do conhecimento. Pesquisa em gestão do conhecimento.

\begin{abstract}
This research aims to identify and to analyze the research themes in Knowledge Management, in order to understand how this scientific field has been constituted and what are the research perspectives within it. For the development of this research, a systematic literature review was carried out using bibliometric studies in Knowledge Management, which were published in the period from 2008 until 2018. The data was grouped into eight themes: nature of knowledge; social, cognitive, and technological dimensions of knowledge; spectrum of knowledge; contexts; areas of interfaces; and studies aimed at the development of the scientific field. The study provides a thematic overview of the Knowledge Management area, pointing out the need to address new issues that relate to contemporary communication challenges. As practical contributions, the results can help researchers, practitioners, and students wishing to study in the field. The results may also serve as a guide for conference organizers to identify topics, as well as for scientific editors to guide future article calls.
\end{abstract}

Keywords: Bibliometric. Knowledge management. Research in knowledge management.

\footnotetext{
1 Universidade UniCesumar, Pró-Reitoria de Pesquisa e Pós-Graduação. Av. Guedner, 1610, Jardim Aclimação, 87050-900, Maringá, PR, Brasil. Correspondência para/Correspondence to: H. P.V.MACHADO. E-mail: <hilkavier@yahoo.com>.

Apoio: Coordenação de Aperfeiçoamento de Pessoal de Nível Superior (PNPD no 88887.339036/2019-00).

Recebido em 19 de maio de 2020, reapresentada em 17 de agosto de 2020 e aprovado em 8 de setembro de 2020.
}

Como citar este artigo/How to cite this article

Machado, H. P. V.; Elias, M. L. G. G. R. Gestão do conhecimento: constituição do campo, temas e perspectivas de pesquisas. Transinformação. v. 32, e200037, 2020. https://doi.org/10.1590/2318-0889202032e200037 


\section{Introdução}

A Gestão do Conhecimento (GC) surgiu, como um campo de estudos, em 1975, com a origem do termo (Serenko; Bontis, 2013; Tzortzaki; Mihiotis, 2014). Ele foi construído inicialmente por profissionais da indústria que buscavam explorar o desenvolvimento do capital intelectual em suas unidades e que, para isso, procuravam gerir o conhecimento (Ma; Yu, 2010; Serenko et al., 2011). Por volta dos anos 90, conferências acadêmicas sobre o tema começaram a atrair pesquisadores de todo o mundo (Serenko et al., 2011). Segundo Ma e Yu, (2010), identificava-se a existência de um campo acadêmico, mas ainda havia certa incerteza sobre suas necessidades e perspectivas futuras.

Desde o início, a GC apresenta interação com outras disciplinas (Ma; Yu, 2010) e a presença de várias disciplinas é uma característica do campo (Serenko, 2013; Qiu; Lv, 2014; Kokol et al., 2015) que, ao mesmo tempo em que amplia perspectivas analíticas, dificulta a compreensão da sua identidade (Serenko, 2013). Com isso, a área não apresenta uma seleção de tópicos que estabeleçam as suas fronteiras (Serenko et al., 2011; Wang et al., 2018), instigando pesquisadores a refletirem sobre essa questão, na medida em que o campo necessita de uma ontologia comum, de definições universais, de uma base teórica integrada e de uma clara direção de pesquisa (Serenko, 2013). Nesse sentido, lança-se aqui um debate provocativo com a finalidade de compreender do que se fala e do que não se fala quando se refere ao campo da GC; ou seja, quais são os temas que constituem o campo? Quais temas podem ser abordados em pesquisas futuras?

Uma das formas para visualização de temas de pesquisas é por meio de estudos bibliométricos (Serenko, 2013). Esse tipo de estudo vem sendo cada vez mais empregado em diferentes domínios científicos para mapear a estrutura de um campo, compondo um panorama sobre temáticas, tendências, instituições e autores (Zupic; Čater, 2015). No entanto, quando analisados isoladamente, eles não propiciam a compreensão da abrangência e das fronteiras do campo, principalmente pelas diferenças relacionadas às bases de dados e periodicidade. Se por um lado existe essa limitação para a análise isolada, a avaliação de um conjunto de bibliometrias pode fornecer evidências e indicar caminhos e percursos dentro de um campo científico que há 40 anos busca se consolidar.

Tendo em conta esses aspectos, o objetivo deste trabalho foi identificar e analisar temas de pesquisas em Gestão do Conhecimento, a fim de compreender como tem se constituído esse campo científico e quais as perspectivas de pesquisas, de modo a auxiliar pesquisadores, profissionais e estudantes que pretendam se inserir no campo. Trata-se de um estudo qualitativo; uma análise sistemática de estudos bibliométricos em GC publicados no período de 2008 a 2018.

\section{Estruturação do campo de gestão do conhecimento}

As primeiras iniciativas em GC ocorreram em 1975 (Serenko, 2013). A expansão da área foi favorecida, nos anos 1990, com o advento da transformação da economia baseada na informação. Para Serenko e Bontis (2013, p. 141, tradução nossa)², a disciplina de GC emergiu como uma resposta "à pressão por maior eficiência de organizações públicas e privadas e o uso efetivo de seu conhecimento". Com isso, outros campos passaram a abordar o conhecimento como um processo sistêmico, importante para assegurar a competitividade de organizações, como, por exemplo, o campo da Estratégia, com a abordagem das capacidades dinâmicas.

A disseminação das ideias em GC foi fortalecida pela primeira conferência sobre o assunto, realizada em Boston em 1993 (Serenko, 2013). Ainda em 1993, um marco importante foi a criação do Knowledge Research Institute, fundado por Karl Wiig, que exerceu papel importante na disseminação de modelos de criação do conhecimento (Grant, 2011). Outro destaque foi a obra de Peter Drucker, de 1993, intitulada "A sociedade pós capitalista", que descreve o papel do conhecimento na sociedade e como ela estava sendo transformada a partir do recurso do conhecimento, além de introduzir a ideia de trabalhadores do conhecimento e de empresas de conhecimento intensivo (Alajmi; Alhaji, 2018). Durante esse período, incluindo o ano de 1994, a disciplina encontrava-se em "estágio embrionário"e 30\% da

$\mathbf{2}$ No original: "to the increasing pressure on private and public organizations to make more efficient and effective use of their knowledge" (Serenko; Bontis, 2013, p. 141). 
produção sobre o tema era concentrada em produção não acadêmica (Serenko; Bontis, 2013). Esse aspecto dividiu o campo até o ano de 1994 (Serenko; Bontis, 2013; Kokol et al., 2015).

Nesse contexto, foram importantes as obras de Nonaka e Takeuchi (1995) e de Davenport e Prusak (1998), ambas reconhecidas como alicerces teóricos do campo (Serenko, 2013). Elas influenciaram a propagação das ideias do campo em outras áreas e assim emergiu o conceito de capital intelectual, legitimado pelo manifesto da Organização para a Cooperação e Desenvolvimento Econômico (Organisation..., 1996), que ressaltava a importância do conhecimento para as nações. No ano seguinte, em 1997, Leif Edvinsson, na Suécia, formulou a primeira declaração de capital intelectual, fornecendo a base para uma nova abordagem e operacionalização do campo (Serenko et al., 2010). Cinco anos após o manifesto da OECD, em junho de 2001, Tomas Stewart, editor da Harvard Business Review, publicou um artigo afirmando que o poder da mente e o capital intelectual estavam se tornando os ativos mais valiosos dos Estados Unidos da América (Serenko et al., 2010; Serenko; Bontis, 2013; Wang et al., 2018).

O campo científico foi sendo fortalecido com a criação de publicações, como o Learning Organization, lançado no ano de 1994 e, o Journal of Knowledge Management, lançado em 1997, e também, ainda em 1997, com a mudança de nome da Review Business Change e Reengineering, que tornou-se Knowledge and Process Management, compondo assim um conjunto de revistas especializadas e de divulgação de pesquisas em GC (Serenko; Bontis, 2013). Mais tarde, outras revistas seriam criadas, como, por exemplo, o Journal of Information \& Knowledge Management, em 2002 (Alajmi; Alhaji, 2018). Outro marco importante ocorreu em 1999, com a introdução da expressão "ativos do conhecimento", cunhada por Boisot (1999).

Analisando a formação do campo, autores identificaram a sua trajetória sob diferentes perspectivas. Por exemplo, Kokol et al. (2015) classificaram cinco momentos distintos da formação do campo: infraestrutura e pesquisa, técnicas e sistemas de GC, tecnologias, aplicações práticas em organizações e avaliação e validação de práticas de GC. No primeiro momento, os tópicos discutidos foram: sistemas gerenciais, bibliotecas digitais, pesquisas orientadas para a área da saúde e pesquisas médicas. Em seguida, os pesquisadores dividiram-se em dois grupos: um que focava em questões técnicas e em como praticar a GC (técnicas, softwares, reuso, captura, acesso, meta dados e conhecimento explícito) e outro que focava nos usuários de GC (trabalhadores, clientes). Na sequência, os estudos passaram a identificar temas abordados nos estudos sobre GC: cultura organizacional, objetivos e redes sociais, além de temas específicos, como: hospitais, organizações de saúde, médias empresas. Segundo os autores, as publicações recentes tratam da avaliação e validação das práticas de GC, métodos e tecnologias.

Por sua vez, para Serenko (2013), o desenvolvimento do campo se dará em quatro gerações: a primeira, que ocorreu em meados dos anos 1990, dava ênfase à gestão e aos processos tecnocêntricos, que buscavam identificar o conhecimento de empregados, codificá-lo e armazená-lo. A segunda geração, de 1990 até início dos anos 2000, reconhecia o valor do capital humano, a transformação de conhecimentos tácitos em explícitos, a organização do capital intelectual e as iniciativas culturais e pessoais. A terceira geração abrangeu os estudos da atualidade e enfatizava perspectivas estratégicas no aprendizado social, inovação social ética, impacto das práticas de GC, desenvolvimento nacional e criação de valor. Já a quarta geração, para o autor, lidará com a complexidade do domínio do conhecimento por meio do desenvolvimento de novas metáforas, paradigmas e ferramentas movidas pela relevância do capital intelectual.

Em outra perspectiva, Tzortzaki e Mihiotis (2014) consideram que a estruturação do campo se deu a partir de três momentos: o primeiro terminou em 1994 com o lançamento de uma obra de Nonaka que trazia uma extensão das teorias de Boisot; o segundo teve início em 1995 com a publicação de Nonaka e Takeuchi (1995) sobre o modelo dinâmico de conhecimento constituído pelas etapas denominadas: socialização, externalização, combinação e internalização do conhecimento e também com a publicação do manifesto da Organização para a Cooperação e Desenvolvimento Econômico (Organisation..., 1996), que favoreceu o surgimento do conceito de capital intelectual. Para os autores, esse segundo momento focava em processos de conhecimento e em espaços colaborativos e de inovação. O terceiro momento teve início em 2001, marcado pela visão do conhecimento como um processo social influenciado por autores como Tsoukas e Vladmirou (2001), Snowden (2002) e Sveiby (2001). A partir do terceiro momento, a abordagem do fenômeno apresentou-se em duas perspectivas: uma que considerava o conhecimento 
um ativo passível de armazenamento, transferência e manipulação, para a qual a GC representava um "processo sistemático de adquirir, organizar, manter, aplicar, compartilhar, e renovar todas as formas de conhecimento, para melhorar o desempenho da organização e criar valor" (Kokol et al., 2015, p. 1, tradução nossa) ${ }^{\mathbf{3}}$ e outra que considerava o conhecimento um processo subjetivo, uma prática ou um estado da mente (Makambe, 2015). Para essa última, o conhecimento representa "um processo dinâmico que transforma uma prática não reflexiva em uma prática reflexiva, elucidando as regras que orientam as atividades da prática, contribuindo para compreensão coletiva e facilitando o surgimento do conhecimento heurístico" (Tsoukas; Valdimirou, 2001, p. 973, tradução nossa) ${ }^{4}$.

Ma e Yu (2010) identificaram dois períodos na trajetória dos estudos: o primeiro, de 1998 a 2002, cujo foco era a busca de definição do campo e da teoria da GC, e o segundo período, de 2003 a 2007, com estudos sobre estratégias de GC, aprendizado organizacional, GC em organização e inovação. Kumar e Mohindra (2015) salientaram que o advento das estruturas de tecnologia da informação influenciou sobremaneira a criação, desenvolvimento, gestão e distribuição do conhecimento. O campo passou, segundo Serenko (2013), a ter duas raízes explicativas: uma soft (a parte humana) e a outra hard (tecnologia da informação).

Além disso, Serenko (2013) identificou que apenas 31,00\% das publicações sobre GC ocorrem em periódicos da área, como o Knowledge Management Journal; 13,00\% em revistas da área de Sistema de Informações Gerenciais; 11,51\% na área de Gestão Geral; 6,65\% em Tecnologia e Inovação e o restante encontra-se disperso em outros 32 campos do conhecimento. Isso significa, segundo o autor, que a GC não é uma disciplina de referência e que estudiosos de GC emprestam ideias de outros campos, evidenciando que o campo de GC carece de um corpo de publicações e temas capazes de propiciar uma visão da sua identidade, ainda que multi e interdisciplinar (Walter; Ribiére, 2013).

Diante do exposto, faz-se necessária uma compreensão de temas e tópicos de estudos que compõem a área da GC. Estudos bibliométricos, como comentado anteriormente, representam uma análise da trajetória de um campo científico, mas é importante realizar uma análise conjunta dos estudos para que se obtenha uma visão geral do campo.

\section{Procedimentos Metodológicos}

Este estudo é uma revisão sistemática de literatura; um método sistematizado de fazer pesquisa a partir de publicações existentes, sintetizando dados anteriormente publicados e propiciando um sentido do "status quo atual da pesquisa" (Kraus; Breier; Dasí-Rodriguez, 2020, p. 6, tradução nossa)5. Os primeiros estudos cientométricos em GC foram publicados no período de 1997 a 2001, tendo se desenvolvido no período de 2002 a 2006 e se consolidado no período de 2007 a 2012 (Serenko, 2013).

A revisão foi realizada de acordo com os parâmetros de Kraus; Breier e Dasí-Rodriguez (2020), seguindo as seguintes etapas: definição da pergunta prévia; definição de um protocolo com os parâmetros da pesquisa (strings de busca, bases de dados, critérios de inclusão e exclusão); extração e síntese dos dados e análise e síntese dos dados. As questões que deram origem ao estudo foram: quais temas constituem o campo da GC? Quais temas pesquisas futuras poderão abordar?

Com relação ao protocolo, foram selecionadas as seguintes bases de dados: Scopus, Web of Science e Scielo (Scientific Eletronic Library Online). Tanto a Scopus quanto a Web of Science são reconhecidas como bases que indexam jornais de qualidade científica (Ellegaard; Wallin, 2015). Já o Scielo tem 20 anos de funcionamento na América Latina, constituindo-se como uma das principais fontes de acesso aberto (Mugnaini; Digiampetri; Mena-Chalco, 2014). Os estudos bibliométricos na forma de artigos científicos em língua inglesa foram selecionados como o tipo de publicação a ser analisada, pois, embora Serenko e Bontis (2013) mencionem estudos em outros idiomas além do inglês (Chinês, Português, Alemão, entre outros), há um predomínio de artigos escritos em língua inglesa

\footnotetext{
3 No original: "processes for acquiring, organizing, sustaining applying, sharing, and renewing all forms of knowledge to enhance the organizational performance and create value" (Kokol et al., 2015, p. 1).

${ }^{4}$ No original: "is the dynamic process of turning an unrefletive practice into a reflective one by elucidating the rules guiding the activities of the practice, by helping give a particular shape to collective understandings, and facilitating the emergence of heuristic knowledge" (Tsoukas; Valdimirou, 2001, p. 973).

5 No original: "status quo of current research" (Kraus; Breier; Dasí-Rodriguez, 2020, p. 6).
} 
na comunicação científica da área, além do fato de que os indicadores cientométicos apontam que os periódicos de maior impacto são veiculados em língua inglesa (Ramy et al., 2018). A busca foi realizada em julho de 2019 e os strings e o número de artigos encontrados em cada uma das bases está detalhado na Tabela 1. O recorte temporal foi de 2008 a 2018, entendendo que pela natureza dos textos estudados, cientometrias/bibliometrias, seus achados englobariam estudos publicados anteriormente e ofereceriam um quadro atualizado de estudos contemporâneos.

Tabela 1. Strings e número de artigos por base de dados.

\begin{tabular}{|c|c|c|}
\hline Base & Strings de busca & Artigos \\
\hline Scopus & $\begin{array}{l}\text { (TITLE-ABS-KEY ("knowledge management") AND TITLE-ABS-KEY ("bibliometric analysis") OR TITLE-ABS- } \\
\text { KEY ("citation analysis") OR TITLE-ABS-KEY ("citation study") OR TITLE-ABS-KEY ("scientometric study") } \\
\text { OR TITLE-ABS-KEY ("scientometric analysis") OR TITLE-ABS-KEY ("publication analysis") OR TITLE-ABS-KEY } \\
\text { ("scholarly impact") AND DOCTYPE (ar) AND PUBYEAR > } 2007 \text { AND PUBYEAR < } 2019\end{array}$ & 85 \\
\hline Scielo & $\begin{array}{l}\text { ("knowledge management") AND ("bibliometric analysis") OR ("citation analysis") OR ("citation study") } \\
\text { OR ("scientometric study") OR ("publication analysis") OR ("scholarly impact") AND la:("pt" OR "en") AND } \\
\text { year_cluster:("2015" OR "2013" OR "2014" OR "2012" OR"2017" OR "2016" OR "2009" OR "2010" OR "2008" OR } \\
\text { "2011") AND is_citable:("is_true") AND type:("research-article") }\end{array}$ & 258 \\
\hline Web of Science & $\begin{array}{l}\text { Você pesquisou por: TÓPICO: ("knowledge management") AND TÓPICO: ("bibliometric analysis") OR } \\
\text { TÓPICO: ("citation analysis") OR TÓPICO: ("citation study") OR TÓPICO: ("scientometric study") OR TÓPICO: } \\
\text { ("scientometric analysis") OR TÓPICO: ("publication analysis") OR TÓPICO: ("scholarly impact") } \\
\text { Refinado por:TÓPICO: ("knowledge management") } \\
\text { Tempo estipulado: 2008-2018. Índices: SCI-EXPANDED, SSCI, A\&HCI, CPCI-S, CPCI-SSH, ESCI. }\end{array}$ & 90 \\
\hline Total & & 433 \\
\hline
\end{tabular}

Fonte: Elaborado pelas autoras (2020).

Nota: A\&HCl: Arts \& Humanities Citation Index; CPCI-S: ConferenceProceedings Citation Index-Science; CPCI-SSH: Conference Proceedings Citation Index Social Sciences \& Humanities; ESCl: Emerging Sources Citation Index; SCI-Expanded: Science Citation Index Expanded; Scielo: Scientific Eletronic Library Online; SSCI: Social Science Citation Index Expanded.

A primeira rodada da pesquisa resultou em 433 artigos, 85 encontrados na plataforma Scopus, 258 no Scielo e 90 na Web of Science, que foram exportados para o software START, utilizado na triagem dos textos. Como critério de inclusão, foram lidos os títulos, resumos e palavras-chave, sendo incluídos os artigos que combinassem "bibliometria", "análise de citação", "análise de publicação", "estudo de impacto" ou "Cientometria" com "Gestão Do Conhecimento".

Em seguida, outro critério instituía a inclusão apenas de pesquisas bibliométricas, análise de citação ou publicações que tratassem de abordagens sobre o campo global da Gestão do Conhecimento. Sessenta e oito dos 433 artigos incialmente encontrados (16\%) foram selecionados. Foram rejeitados 16 artigos que estavam duplicados e os que abordavam a bibliometria em tópicos específicos ou em outros campos (349 artigos). Os 68 artigos selecionados foram submetidos a uma segunda rodada de avaliação, na qual os resumos foram analisados por ambas as pesquisadoras. Foram então excluídos artigos que tratavam de casos específicos e/ou focavam em outros aspectos teóricos que não o campo da GC. Com isso, restaram 24 artigos, sendo que 22 deles foram publicados em periódicos científicos e dois foram apresentados em congressos e indexados nos portais consultados. Por meio do Google Scholar identificou-se que um dos trabalhos foi publicado em periódicos posteriormente e, a fim de aprimorar a análise, utilizou-se a versão publicada em periódico. Outra substituição foi em relação ao artigo de Serenko e Bontis (2017), que substituiu a versão anterior, publicada em 2012.

As 24 publicações selecionadas foram examinadas por meio da análise temática (Maguire; Delahunt, 2017) que consiste em identificação e análise de padrões (temas) dos dados, organizando-os e descrevendo-os em detalhes. A análise temática é um método desenvolvido em cinco etapas. A primeira delas consiste na familiarização com os dados. Nessa etapa, ambas as autoras leram todos os artigos e trocaram impressões a respeito das publicações. Na segunda etapa, que consiste na codificação dos dados, os códigos foram gerados a partir de um critério semântico, utilizando-se o conteúdo explícito dos dados. Com isso, os códigos foram gerados a partir dos temas abordados pelos autores e estão detalhados no Quadro 2. As etapas três, quatro e cinco constituem, respectivamente, na busca, revisão e definição de temas. As pesquisadoras examinaram os temas abordados e codificados a fim de identificarem 
similaridades. Foram então definidos oito temas, posteriormente revisados e nominados pelas autoras. A sexta etapa consiste na descrição dos temas. Todos os códigos foram alocados em temas e apresentados no Quadro 3, na coluna subtemas.

\section{Resultados}

O Quadro 1 apresenta o detalhamento dos estudos consultados, em ordem de ano de publicação. Observase que o autor que mais publicou estudos bibliométricos foi Alexandre Serenko, vinculado a uma universidade canadense. A maior quantidade de artigos (oito) foi publicada no periódico Journal of Knowledge Management. Um estudo foi publicado em periódico nacional (Transinformação), em 2013. Os estudos bibliométricos foram distribuídos ao longo do período de 2010 a 2018, sendo que no último ano foram publicados cinco estudos - o que evidencia a busca pela compreensão da trajetória e dos limites do campo de estudos em GC.

Quadro 1. Detalhamento dos estudos bibliométricos.

\begin{tabular}{|c|c|}
\hline Autores & Detalhamento do estudo \\
\hline Bontis; Serenko (2009) & $\begin{array}{l}\text { O estudo teve como objetivo desenvolver um ranking de Journals in Knowledge Management e capital } \\
\text { intelectual. }\end{array}$ \\
\hline Ma; Yu (2010) & $\begin{array}{l}\text { O estudo faz uma análise de citação, co-citação entre outros de artigos publicados entre } 1998 \text { e } 2007, \\
\text { usando a palavras chave "Knowledge management". }\end{array}$ \\
\hline Serenko et al. (2010) & $\begin{array}{l}\text { Os autores analisaram os temas Gestão do conhecimento e capital intelectual publicados no período } 1994 \\
\text { e } 2008 \text { em onze periódicos das áreas. }\end{array}$ \\
\hline Serenko et al. (2011) & Foco na análise do número de artigos publicados por autor na área de Gestão do conhecimento. \\
\hline Wallace; Van Fleet; Downs (2011) & O estudo focou metodologias utilizadas em 20 Journals em Gestão do conhecimento. \\
\hline Uzunboylu; Eriş; Ozcinar (2011) & O estudo analisou a presença da gestão do conhecimento na área de Educação. \\
\hline Dwivedi et al. (2011) & Autores analisaram publicações em gestão do conhecimento no período 1974-2008. \\
\hline Curado; Oliveira; Maçada (2011) & $\begin{array}{l}\text { Os autores verificaram publicações em gestão do conhecimento nos journals: Information and Management, } \\
\text { Information processing and management, International journal of Information management, Journal of Global } \\
\text { Information Management, Journal of Management Information Systems, Management Information Systems } \\
\text { Quarterly. }\end{array}$ \\
\hline Grant (2011) & Estudo bibliométrico da gestão do conhecimento no período 1990 a 2009. \\
\hline Lee; Chen (2012) & Estudo bibliométrico da área de gestão do conhecimento com publicações no período de 1995 a 2010. \\
\hline Serenko (2013) & Meta análise com 108 estudos cientométricos em gestão do conhecimento. \\
\hline Madeira; Vick; Nagano (2013) & $\begin{array}{l}\text { Foco na relação entre Gestão do conhecimento, gestão da inovação, gestão de tecnologia e gestão da } \\
\text { informação com publicações no período de 2006-2012. }\end{array}$ \\
\hline Serenko; Bontis (2013) & $\begin{array}{l}\text { Analisaram os artigos mais influentes na área de gestão do conhecimento publicados no Journal of } \\
\text { Knowledge Management. }\end{array}$ \\
\hline Walter; Ribière (2013) & Análise de citação e co-citação das publicações do Knowledge Management Research and Practice. \\
\hline Qiu; Lv (2014) & Estudo com foco em publicações em gestão do conhecimento na base web of Science, período 1993 a 2012. \\
\hline Kokol et al. (2015) & $\begin{array}{l}\text { Estudo com foco em publicações sobre gestão do conhecimento na área de Administração, utilizando a } \\
\text { base de dados da Scopus e o período de 1996-2010. }\end{array}$ \\
\hline Khasseh; Mokhtarpour (2016) & Foco na análise de citação em gestão do conhecimento. \\
\hline Akhavan et al. (2016) & Estudo sobre os 500 artigos mais citados no campo de Gestão do conhecimento, no período 1980 e 2014. \\
\hline Serenko; Bontis (2017) & Estudo bibliométrico atualizando estudo anterior dos autores referente ao período 2008 a 2012. \\
\hline Wang et al. (2018) & $\begin{array}{l}\text { Avaliação das publicações em gestão do conhecimento com base na base Web of Science e no período } \\
\text { de 1974-2017. }\end{array}$ \\
\hline Gaviria-Marin; Merigo; Popa (2018) & Estudo sobre as publicações no Journal of Knowledge Management no período de 1997 a 2016. \\
\hline Alajmi; Alhaji (2018) & Estudo sobre as publicações no Journal of Information \& Knowledge Management (JIKM) no período 2002-2016. \\
\hline Ramy et al. (2018) & Estudo das publicações no Knowledge Management Research and Practice (KMRP) no período 2003-2015. \\
\hline Goswami; Agrawal (2018) & Estudo bibliométrico sobre compartilhamento de conhecimento. \\
\hline
\end{tabular}

Fonte: Elaborado pelas autoras (2020). 
Com base na leitura dos textos, foi realizada a codificação dos temas em GC referenciados nos artigos, salientando que alguns deles não traziam temas, mas apenas análise de co-citação. O Quadro 2 apresenta uma síntese de 27 temas identificados nos estudos consultados.

Quadro 2. Relação entre temas abordados e autores.

\begin{tabular}{|c|c|}
\hline Temas & Autores \\
\hline Processos & $\begin{array}{l}\text { Dwivedi et al. (201 1); Grant (2011); Kokol et al. (2015); Alajmi; Alhaji } \\
\text { (2018); Gaviria-Marin et al. (2018). }\end{array}$ \\
\hline Tipos de conhecimento & Akhavan et al. (2016). \\
\hline Informação e Sistemas & $\begin{array}{l}\text { Dwivedi et al. (2011); Grant (2011); Qiu; Lv (2014); Akhavan et al. (2016); } \\
\text { Gaviria-Marin et al. (2018); Wang et al. (2018). }\end{array}$ \\
\hline Capital Intelectual & $\begin{array}{l}\text { Grant (2011); Lee; Chen (2012); Walter; Ribiére, (2013); Qiu; Lv (2014); } \\
\text { Gaviria-Marin et al. (2018); Ramy et al. (2018); Wang et al. (2018). }\end{array}$ \\
\hline Aprendizagem e comunidades de prática & $\begin{array}{l}\text { Ma; Yu (2010); Grant (2011); Walter; Ribiére (2013); Qiu e Lv (2014); } \\
\text { Khasseh; Mokhtarpour (2016); Gaviria-Marin et al. (2018); Ramy et al. } \\
\text { (2018); Wang et al. (2018). }\end{array}$ \\
\hline Criação e Aquisição de conhecimento, capacidade absortiva & $\begin{array}{l}\text { Lee; Chen (2012); Qiu; Lv (2014); Kokol et al. (2015); Akhavan et al. } \\
\text { (2016); Ramy et al. (2018); Wang et al. (2018). }\end{array}$ \\
\hline Compartilhamento de conhecimento & $\begin{array}{l}\text { Lee; Chen (2012); Qiu; Lv (2014); Kokol et al. (2015); Akhavan et al. } \\
\text { (2016); Gaviria-Marin et al. (2018); Ramy et al. (2018); Wang et al. (2018). }\end{array}$ \\
\hline Transferência de conhecimento & $\begin{array}{l}\text { Lee; Chen (2012); Qiu; Lv (2014); Akhavan et al. (2016); Gaviria-Marin et } \\
\text { al. (2018); Ramy et al. (2018). }\end{array}$ \\
\hline Conversão e socialização do conhecimento & Kokol et al. (2015). \\
\hline Reutilização do conhecimento & Lee; Chen (2012). \\
\hline Networks/Redes Sociais e Capital Social- & $\begin{array}{l}\text { Lee; Chen (2012); Walter; Ribiére, (2013); Kokol et al. (2015); Khasseh; } \\
\text { Mokhtarpour (2016); Ramy et al. (2018); Wang et al. (2018). }\end{array}$ \\
\hline Memória organizacional & Lee; Chen (2012). \\
\hline Modelos & Grant (2011); Lee; Chen (2012); Kokol et al. (2015); Akhavan et al. (2016). \\
\hline Planejamento, Políticas, Avaliação e Estratégias de Conhecimento & Ma; Yu (2010); Dwivedi et al. (2011); Grant (2011); Akhavan et al. (2016). \\
\hline Pesquisa e Métodos de Pesquisa & Dwivedi et al. (2011); Walter; Ribiére (2013). \\
\hline Conhecimento e Inovação & $\begin{array}{l}\text { Ma; Yu (2010); Lee; Chen (2012); Qiu; Lv (2014); Khasseh; Mokhtarpour } \\
\text { (2016); Gaviria-Marin et al. (2018); Ramy et al. (2018); Wang et al. (2018). }\end{array}$ \\
\hline Cultura & $\begin{array}{l}\text { Kokol et al. (2015); Akhavan et al. (2016); Gaviria-Marin et al. (2018); } \\
\text { Ramy et al. (2018); Wang et al. (2018). }\end{array}$ \\
\hline Inteligência organizacional & Lee; Chen (2012). \\
\hline Web Semântica & Qiu; Lv (2014); Kokol et al. (2015); Wang et al. (2018). \\
\hline Data Mining & Qiu; Lv (2014); Wang et al. (2018). \\
\hline Metadados & Kokol et al. (2015). \\
\hline Engenharia de Software & Kokol et al. (2015); Alajmi e Alhaji (2018). \\
\hline Inteligência competitiva & Kokol et al. (2015). \\
\hline E-learning & Qiu; Lv (2014); Kokol et al. (2015). \\
\hline Gestão da Informação & $\begin{array}{l}\text { Qiu; Lv (2014); Kokol et al. (2015); Gaviria-Marin et al. (2018); Wang et al. } \\
\text { (2018). }\end{array}$ \\
\hline Gestão de Projetos & Qiu; Lv (2014); Kokol et al. (2015). \\
\hline Saúde e Educação & Kokol et al. (2015). \\
\hline
\end{tabular}

Fonte: Elaborado pelas autoras (2020).

Observa-se nos dados do Quadro 2 que os seguintes temas foram mencionados por um autor apenas: tipos de conhecimento; conversão, socialização e reutilização do conhecimento; memória e inteligência organizacional; inteligência competitiva; saúde e educação e meta dados. Nota-se também que alguns temas aparecem de forma mais 
constante nos estudos, tais como: processos de conhecimento, informação e sistemas; capital intelectual; aprendizagem e comunidades de prática; criação e aquisição de conhecimento; capacidade absortiva; compartilhamento e transferência de conhecimento; modelos; conhecimento e inovação; gestão da informação e gestão de projetos.

Na sequência, os temas identificados no Quadro 2 foram categorizados em oito subtemas, apresentados no Quadro 3. Os quatro temas iniciais abrangem aspectos associados às dinâmicas sociais, cognitivas e tecnológicas do conhecimento, bem como à natureza do conhecimento. No tema "espectro do conhecimento" agrupam-se os subtemas associados às faces de análise da Gestão do Conhecimento. O tema "contextos" refere-se às possiblidades de aplicação do conceito em diferentes domínios. O tema "áreas de interfaces" abrange outras áreas de conhecimento que estudam GC, conforme os dados dos estudos bibliométricos, conforme os dados dos estudos bibliométricos. Por fim, o último tema versa sobre estudos voltados ao desenvolvimento do campo científico.

Quadro 3. Categorização dos temas.

\begin{tabular}{|c|c|}
\hline Categoria & Temas \\
\hline \multirow{2}{*}{ Natureza do Conhecimento } & Tipos de conhecimento \\
\hline & Processos \\
\hline \multirow{4}{*}{ Dimensão Social/individual } & Capital Intelectual \\
\hline & Capital social \\
\hline & Redes \\
\hline & Comunidades de Prática \\
\hline \multirow{3}{*}{ Tecnologia voltada à produção e armazenamento de dados } & Web Semântica \\
\hline & Data Mining \\
\hline & Metadados \\
\hline \multirow{3}{*}{ Dimensão Cognitiva } & E-learning \\
\hline & Aprendizagem \\
\hline & Memória \\
\hline \multirow{9}{*}{ Espectro do conhecimento } & Planejamento, políticas e estratégias de conhecimento \\
\hline & Informação \\
\hline & Modelos \\
\hline & Criação e Aquisição de Conhecimento \\
\hline & Compartilhamento \\
\hline & Transferência \\
\hline & Conversão/Socialização \\
\hline & Reutilização \\
\hline & Avaliação do conhecimento \\
\hline \multirow[t]{4}{*}{ Contextos } & Saúde, Educação \\
\hline & Gestão da Informação \\
\hline & Gestão de Projetos \\
\hline & Engenharia de Software \\
\hline \multirow[t]{4}{*}{ Áreas de interfaces } & Estratégia (inteligência competitiva, capacidade absortiva) \\
\hline & Inovação \\
\hline & Estudos Organizacionais (Cultura, inteligência organizacional) \\
\hline & Sistemas de informação \\
\hline Desenvolvimento científico & Pesquisa e Métodos de Pesquisa \\
\hline
\end{tabular}

Fonte: Elaborado pelas autoras (2020). 


\section{Discussão}

A codificação dos temas apresentados nos estudos bibliométricos em GC consultados durante esta pesquisa evidencia que alguns temas se estabeleceram, a partir dos anos 1990, como centrais no campo de estudos de Gestão do Conhecimento. Dentre eles, destaca-se o tema "compartilhamento e transferência do conhecimento" (Lee; Chen, 2012; Ramy et al., 2018). Desde 1987 são publicados estudos sobre modelos de conhecimento e sistemas, sendo que até 2006 houve um aumento no número de publicações com essas temáticas (Grant, 2011; Lee; Chen, 2012).

O tema "criação do conhecimento", embora discutido desde 1995 foi o que apareceu de forma menos frequente nos estudos analisados (Lee; Chen, 2012; Qiu; Lv, 2014; Ramy et al., 2018). Além do tema "criação", outros que apareceram menos frequentemente foram: "sistemas de informação" e "aprendizado organizacional" (Ramy et al., 2018). Já o número de publicações que abordaram os temas "gestão da informação" e"tecnologia da informação" diminuiu (Qiu; Lv, 2014).

Entre os temas que cresceram em número de publicações e que apareceram de forma mais frequente estão: "capital intelectual" e"capacidade absortiva" (Qiu; Lv, 2014; Alajmi; Alhaji, 2018; Ramy et al., 2018). Entre os temas que começaram a aparecer recentemente estão:"reutilização do conhecimento", "inteligência organizacional","engenharia de software" e, a partir de 2016, "big data" (Lee; Chen, 2012).

É importante salientar que temas como "aprendizado","projetos","capital intelectual" e "capacidade absortiva”, por exemplo, estão entre os mais abordados no campo, porém têm sido discutidos também em outras áreas, sendo considerados "temas fronteiras" (Grant, 2011; Alajmi; Alhaji, 2018). O mesmo acontece com os temas "inovação" e "cultura" (Ramy et al., 2018).

A categorização apresentada no Quadro 3 retrata uma visão do campo de pesquisas em GC, evidenciando quais foram os temas discutidos em cada categoria. Com isso, é possível ter uma visão do que se discute quando se refere à GC como campo de estudos. Isso contribui para a compreensão da identidade científica de um campo que é permeado por várias áreas de estudos e que influencia outros campos. A esse respeito, vale citar o conceito de Gestão Estratégica do Conhecimento, que abrange a criação de conhecimento como um ativo estratégico por meio de alianças estratégicas e capital intelectual (Ferreira; Mueller; Papa, 2020).

Ademais, as categorias apresentadas no Quadro 3 abrangem diferentes possibilidades de pesquisas a partir dos temas identificados, os quais podem contribuir para que o campo da GC se fortaleça e crie uma identidade própria. Com isso, o campo avança no sentido de construir uma base teórica própria, o que Serenko (2013) afirma ser uma necessidade.

Outro objetivo deste trabalho era apontar perspectivas para futuras pesquisas. Nesse sentido, um dos aspectos mencionado por alguns estudos consultados refere-se à necessidade de que sejam publicados trabalhos que apresentem uma visão crítica de GC. Considera-se que essa visão crítica é importante para a compreensão do conhecimento como um constructo que resulta de um processo dinâmico e coletivo, como citado por Tsoukas e Vladimirou (2001). Além disso, Serenko (2013) sugere a necessidade de estudos voltados aos temas "consequências negativas de GC", "princípios de desaprender" e "organizações não lucrativas".

Não foram identificados estudos críticos, os quais poderiam ser importantes para promover uma avaliação de práticas e estratégias de pesquisa e de ação. Faltaram também estudos com temas associados à dinâmica do conhecimento e à informação na contemporaneidade, tal como "conhecimento em espaços de trabalho compartilhados" (Bouncken; Alsam, 2019). Há um campo para pesquisas sobre a digitalização da sociedade, abrangendo transformações em práticas informacionais que afetam a produção, a circulação e o consumo de informações e as formas de mobilização social (Escande-Gauguié; Naivin, 2019). A sociedade atual marcha em alta velocidade e isso está também associado aos fluxos informacionais simultâneos que requerem a gestão das informações presente, futura e passada e que expressem o que se denomina conhecimento na atualidade.

Nesse sentido, o campo da GC tem dado pouca ênfase às discussões associadas à dinâmica da informação na sociedade, como a"pós-verdade" (Oxford, 2016), que se refere às circunstâncias nas quais os fatos objetivos apresentam 
menor importância para a formação da opinião pública do que as avaliações ou opiniões pessoais ou ainda as fake news, expressão que teve origem em 2016 e que configura a partilha deliberada de informações falsas. Nesses casos, a informação visa influenciar a formação da opinião pública abrangendo falsidade da informação e intencionalidade negativa e distinguindo-se da anti-informação e da informação incorreta (Allard-Huver, 2019).

\section{Conclusão}

O objetivo deste trabalho foi identificar e analisar temas de pesquisas em Gestão do Conhecimento que propiciassem a compreensão de como esse campo científico tem se constituído e quais as perspectivas de investigações. A análise dos estudos bibliométricos demonstrou que o campo tem se estabelecido de modo inter e multidisciplinar, o que torna o estudo atraente, mas, ao mesmo tempo, contribui para um conceito de GC multi facetado e difícil de capturar. Por meio da análise temática, foram identificadas oito categorias que representam "do que se fala" e "quando se fala"em GC, sendo: natureza do conhecimento; dimensão social/individual; tecnologia voltada à produção e armazenamento de dados; dimensão cognitiva; espectro do conhecimento; contexto; áreas de interfaces e desenvolvimento do campo científico.

Quanto às perspectivas de estudos futuros, de acordo com os dados obtidos através desta pesquisa são necessários estudos críticos, além de enfoques associados à dinâmica da informação na sociedade contemporânea, como a pós-verdade e as fake news, por exemplo. Um dos autores sugere a necessidade de estudos que abordem princípios do desaprender e, nesse sentido, enquadram-se também estudos sobre esquecimento organizacional.

Como contribuição teórica, este estudo representa um avanço em relação aos estudos anteriores pela profundidade da análise e pela categorização dos temas, bem como pela avaliação de tópicos ausentes nas publicações relacionadas ao campo. Os resultados oferecem um panorama temático do campo da Gestão do Conhecimento, apontando a necessidade de abordar novos temas que estejam atentos aos desafios comunicacionais contemporâneos. Além disso, o artigo oferece uma contribuição metodológica ao fazer uma revisão sistemática de bibliometrias.

Como contribuições práticas, os resultados podem auxiliar pesquisadores, profissionais e estudantes que pretendam se inserir no campo. Os resultados poderão ainda orientar organizadores de conferências e editores científicos.

Como limitações do artigo, destaca-se que foram analisadas bibliometrias em Gestão do Conhecimento; porém, considerando que o assunto tem sido discutido em tópicos abordados em outras áreas, estudos futuros podem identificar temas relacionados à Gestão de Conhecimento que estejam sendo discutidos em outros campos.

\section{Agradecimentos}

As autoras agradecem aos revisores anônimos, pelas relevantes contribuições para melhoria do artigo.

\section{Colaboradores}

H. P.V. MACHADO e M. L. G. G. R. ELIAS participaram em conjunto da concepção e desenho da pesquisa, bem como da análise e interpretação dos dados. A versão final do artigo também contou com a revisão e aprovação de ambas as autoras. 
Akhavan, P. et al. Major trends in knowledge management research: a bibliometric study. Scientometrics, v. 107, n. 3, p. 12491264, 2016. Doi: http://dx.doi.org/10.1007/s11192-016-1938-x.

Alajmi, B; Alhaji, T. Mapping the field of knowledge management: bibliometric and content analysis of Journal of Information \& Knowledge Management for the period from 2002-2016. Journal of Information \& Knowledge Management, v. 17, n. 3, p. 1850027 , 2018. Doi: http://dx.doi.org/10.1142/S0219649218500272.

Allard-Huver, F. Fake news. In: Escande-Gauguié, P.; Naivin, B. (org.). Comprendre la culture numérique. Paris: Dunod, 2019.

Boisot, M. Knowledge assets securing competitive advantage in the information economy. New York: Oxford University Press, 1999.

Bontis, N.; Serenko, A. A follow-up ranking of academic journals. Journal of Knowledge Management, v. 13, n. 1, p. 16-26, 2009. Doi: 10.1108/13673270910931134

Bouncken, R.; Aslam. M. M. Understanding knowledge exchange processes among diverse users of coworking-spaces. Journal of Knowledge Management, v. 23, n. 10, p. 2067-2085, 2019. Doi: htttp://dx.doi.org/10.1108/JKM-05-2018-0316.

Curado, C.; Oliveira, M., Maçada, A. C. G. Mapping knowledge management authoring patterns and practices. African Journal of Business Management, v. 5 n. 22, p. 9137-9153, 2011. Doi: htttp://dx.doi.org/11.5897/ajbm11.163.

Davenport, T. H.; Prusak, L. Working knowledge: how organizations manage what they know. [s.l.]: Harvard Business Press, 1998.

Dwivedi, Y. K. et al. Research trends in knowledge management: analyzing the past and predicting the future. Information Systems Management, v. 28, n. 1, p. 43-56, 2011. Doi: https://doi.org/10 .1080/10580530.2011.536112.

Ellegaard, O.; Wallin, J. A. The bibliometric analysis of scholarly production: how great is the impact? Scientometrics, v. 105, n. 3, p. 1809-1831, 2015. Doi: https://doi.org/10.1007/s11192015-1645-Z.

Escande-Gauguié, P.; Naivin B. Comprendre la culture numérique. Paris: Dunod, 2019.

Ferreira, J.; Mueller, J.; Papa, A. Strategic knowledge management: theory, practice and future challenges. Journal of Knowledge Management, v. 24, n. 2, p. 121-126, 2020. Doi: https://doi. org/10.1108/JKM-07-2018-0461.

Gaviria-Marin, M.; Merigo, J. M.; Popa, S. Twenty years of the Journal of Knowledge Management: a bibliometric analysis. Journal of Knowledge Management, v. 22, n. 8, p. 1655-1687, 2018. Doi: 10.1108/JKM-10-2017-0497.

Goswami, A. K.; Agrawal, R. K. A reflection on knowledge sharing research: patterns and trends. Vine Journal of information and Knowledge Management Systems, v. 48, n. 3, p. 352-372, 2018. Doi: https://doi.org/10.1108/VJIKMS-08-2017-0049.

Grant, K. Knowledge management, an enduring but confusing fashion. Electronic Journal of Knowledge Management, v. 9, n. 2, p. 117-131, 2011. Available form: file:///C:/Users/hilka/Downloads/ EJKM-volume-9-issue-2.pdf. Access on: Mar. 18, 2020.
Khasseh, A. A.; Mokhtarpour, R. Tracing the historical origins of knowledge management issues through Referenced Publication Years Spectroscopy (RPYS). Journal of Knowledge Management, v. 20, n. 6, p. 1393-1404, 2016. Doi: https://doi.org/10.1108/ JKM-01-2016-0019.

Kokol, P. et al. Knowledge management in organizations: a bibliometric analysis of research trends. In: Knowledge Management in Organizations, 2015, Maribor. Proceeding [...]. Maribor: Springer International Publishing, 2015

Kraus, S.; Breier, M.; Dasí-Rodriguez, S. The art of crafting a systematic literature review in entrepreneurship research. International Entrepreneurship and Management Journal, v. 16, p. 1023-1042, 2020. Doi: https://doi.org/10.1007/s11365-02000635-4.

Kumar, A.; Mohindra, R. Bibliometric analysis on knowledge management research. International Journal of Information Dissemination and Technology, v. 5, n. 2, p. 106-113, 2015. Available from: https://www.researchgate.net/publication/280940714_ Bibliometric_Analysis_on_Knowledge_Management_Research. Aceess on: Mar. 25, 2019.

Lee, M. R.; Chen, T. T. Revealing research themes and trends in knowledge management: from 1995 to 2010. Knowledge-Based Systems, v. 28, p. 47-58, 2012. Doi: https://doi.org/10.1016/j. knosys.2011.11.016.

Ma, Z; Yu, K. Research paradigms of contemporary knowledge management studies: 1998-2007. Journal of Knowledge Management, v. 14, n. 2, p. 175-189, 2010. Doi: https://doi. org/10.1108/13673271011032337.

Madeira, L. M. M.; Vick, T. E.; Nagano, M. S. Directions of scientific literature in knowledge management from the perspective of their relationships with innovation, information and technology management. Transinformação, v. 25, n. 2, p. 167-174, 2013. Doi: http://dx.doi.org/10.1590/S0103-37862013000200008.

Maguire, M.; Delahunt, B. Doing a thematic analysis: a practical, step-by-step guide for learning and teaching scholars. All Ireland Journal of Higher Education, v. 9, n. 3, p. 3351-33514, 2017. Available from: https://ojs.aishe.org/index.php/aishe-j/ article/view/335. Access on: Sept. 20, 2019.

Mugnaini, R.; Digiampetri, L. A.; Mena-Chalco, J. P. Comunicação científica no Brasil (1998-2012): indexação, crescimento, fluxo e dispersão. Transinformação, v. 26, n. 3, p. 239-252, 2014. Doi: http://dx.doi.org/10.1590/0103-3786201400030002.

Makambe, U. Perspectives on knowledge management: a literature review. Information and Knowledge Management, v. 5, n. 1, p. 88-97, 2015.

Nonaka, I.; Takeuchi, H. The knowledge-creating company: how japanese companies create the dynamics of innovation. [s.l.]: Oxford University Press, 1995.

Organisation for Economic Co-Operation and Development. The knowledge-based economy. Paris: OCDE, 1996. Available from: http://www.oecd.org/ officialdocuments/publicdisplaydocumentpdf/?cote=OCDE/ GD\%2896\%29102\&docLanguage=En. Access on: Nov. 19, 2019. 
Oxford Dictionaries. Word of the year 2016. Oxford: Oxford University Press, 2016. Available from: https://languages.oup. com/word-of-the-year/word-of-the-year-2016. Access on: July 22, 2019.

Qiu, J.; Lv, H. An overview of knowledge management research viewed through the web of science (1993-2012). Aslib Journal of Information Management, v. 66, n. 4, p. 424-442, 2014. Doi: http://dx.doi.org/10.1108/AJIM-12-2013-0133.

Ramy, A. et al. A scientometric analysis of knowledge management research and practice literature: 2003-2015. Knowledge Management Research and Practice, v. 16, n. 1, p. 6677,2018. Doi: http://dx.doi.org/10.1080/14778238.2017.1405776.

Serenko, A. et al. A scientometric analysis of knowledge management and intellectual capital academic literature (1994-2008). Journal of Knowledge Management, v. 14, n. 1, p. 3-23, 2010. Doi: https://doi.org/10.1108/13673271011015534.

Serenko, A. et al. The superstar phenomenon in the knowledge management and intellectual capital academic discipline. Journal of Informetrics, v. 5, n. 3, p. 333-345, 2011. Doi: http:// dx.doi.org/10.1016/j.joi.2011.01.005.

Serenko, A. Meta-analysis of scientometric research of knowledge management: discovering the identity of the discipline. Journal of Knowledge Management, v. 17, n. 5, p. 773-812, 2013. Doi: http://dx.doi.org/10.1108/JKM-05-2013-0166.

Serenko, A.; Bontis, N. Global ranking of knowledge management and intellectual capital academic journals: 2017 update. Journal of Knowledge Management, v. 21, n. 3, p. 675-692, 2017. Doi: https://doi.org/10.1108/JKM-11-2016-0490.

Serenko, A.; Bontis, N. The intellectual core and impact of the knowledge management academic discipline. Journal of Knowledge Management, v. 17, n. 1, p. 137-155, 2013. Doi: https:// doi.org/10.1108/13673271311300840.
Snowden, D. Complex Acts of Knowing: Paradox and Descriptive Self-awareness. Journal of Knowledge Management, v. 6, n. 2, p. 100-111, 2002. Doi: https://doi.org/10.1108/13673270210424639

Sveiby, K. E. What is knowledge Management. Helsinki: Sveiby Associados, 2001.

Tsoukas, H.; Vladimirou, E. What is organizational knowledge? Journal of Management Studies, v. 38, n. 7, p.973-993, 2001. Doi: https://doi.org/10.1111/1467-6486.00268.

Tzortzaki, A. M.; Mihiotis, A. A review of knowledge management theory and future directions. Knowledge and Process Management, v. 21, n. 1, p. 29-41, 2014. Doi: https://doi.org/10.1002/kpm.1429.

Uzunboylu, H.; Eriş, H.; Ozcinar, Z. Results of a citation analysis of knowledge management in education. British Journal of Educational Technology, v. 42, n. 3, p. 527-538, 2011. Doi: https:// doi.org/10.1111/j.1467-8535.2009.01018.x.

Wallace, D. P.; Van Fleet, C.; Downs, L. J. The research core of the knowledge management literature. International Journal of Information Management, v. 31, n. 1, p. 14-20, 2011. Doi: https:// doi.org/10.1016/j.ijinfomgt.2010.10.002.

Walter, C.; Ribière, V. A citation and co-citation analysis of 10 years of KM theory and practices. Knowledge Management Research and Practice, v. 11, n. 3, p. 221-229, 2013. Doi: https:// doi.org/10.1057/kmrp.2012.64.

Wang, P. et al. Visualizing the Academic Discipline of Knowledge Management. Sustainability, v. 10, n. 3, p. 682, 2018. Doi: https:// doi.org/10.3390/su10030682.

Zupic, I.; Čater, T. Bibliometric methods in management and organization. Organizational Research Methods, v. 18, n. 3, p. 429-472, 2015. Doi: https://doi.org/10.1177/1094428114562629. 